\title{
Regenerative medicine of cornea by cell sheet engineering using temperature-responsive culture surfaces
}

\author{
UMEMOTO Terumasa $^{1}$, YAMATO Masayuki $^{1}$, NISHIDA Kohji $^{2} \&$ OKANO Teruo ${ }^{1 *}$ \\ ${ }^{1}$ Institute of Advanced Biomedical Engineering and Science, Tokyo Women's Medical University, Tokyo 162-8666, Japan; \\ ${ }^{2}$ Department of Ophthalmology, Osaka University Medical School, Osaka 565-0871, Japan
}

Received September 28, 2012; accepted December 5, 2012; published online May 22, 2013

\begin{abstract}
Recently, regenerative medicine has been focused on as next-generation definitive therapies for several diseases or injuries for which there are currently no effective treatments. These therapies have been rapidly developed through the effective fusion between different fields such as stem cell biology and biomaterials. So far, we have proposed "cell sheet engineering" through our core technology that simply applies alterations of the temperature which allows regulation of the attachment or detachment of living cells on the culture surfaces grafted with the temperature-responsive polymer "poly( $N$-isoproplyacrylamide)". This technology enables us to construct bioengineered sheet-like tissues, termed "cell sheets", without the need of using biodegradable scaffolds and protease treatments that are traditionally used. Therefore, our carrier-free cell sheets not only are independent of the issues observed in conventional methods, but also showed further advantages in the reconstruction of the corneal epithelium or endothelium (e.g. improvement of optical transparency and cell-cell interactions to host stroma in reconstructed tissues). Moreover, our approach does not have issues such as immune rejection or limited number of donors, due to the use of cell sheets derived from autologous limbal (in patients with unilateral disease) or oral mucosal epithelial cells (in patients with bilateral disorders). Indeed, we have successfully performed the clinical application for the reconstruction of ocular surfaces through the transplantation of our carrier-free corneal epithelial cell sheets, as evidenced by improved visual acuity as well as long-term maintenance of postoperative health conditions on ocular surfaces in all patients. We have also proposed a novel approach for the reconstruction of the corneal endothelium using corneal endothelial cell sheets by demonstrating its successful application. Thus, our cell sheet engineering technique provides a breakthrough in the field of regenerative medicine applied to the cornea.
\end{abstract}

cell sheet, temperature-responsive, carrier-free

Citation: Umemoto T, Yamato M, Nishida K, et al. Regenerative medicine of cornea by cell sheet engineering using temperature-responsive culture surfaces. Chin Sci Bull, 2013, 58: 4349-4356, doi: 10.1007/s11434-013-5742-1

The eye is a crucial organ to get light information from the surrounding environment via its visual performance, and is undoubtedly essential to maintain our quality of life. The cornea is a transparent tissue, which resides most anteriorly in the eye, and is composed of 3 layers (i.e. the epithelial layer, stromal layer, and endothelial layer) (Figure 1). The layers of the epithelium and endothelium in the cornea are mainly composed of only corneal epithelial cells and endothelial cells, respectively. In contrast, the stromal layer contains stromal cells such as keratocytes and massive connective tissue, including collagen. In fact, the interaction be-

*Corresponding author (email: tokano@abmes.twmu.ac.jp) tween these 3 layers accurately governs light refraction in the cornea through the maintenance of both its optical transparency and thickness, which is critical for image formation at the retina. Therefore, loss of corneal transparency by severe diseases or injures induces extremely impaired visual performance. To restore corneal transparency in such a damaged cornea, transplantation of an allogeneic cornea supplied by the Eye Bank has been traditionally performed. However, transplantation therapies have been greatly limited by the serious shortage of donors or immune rejection. To fundamentally overcome these issues in transplantation medicine, the applications of artificial corneas were previously attempted as an alternative cure, but have not been 


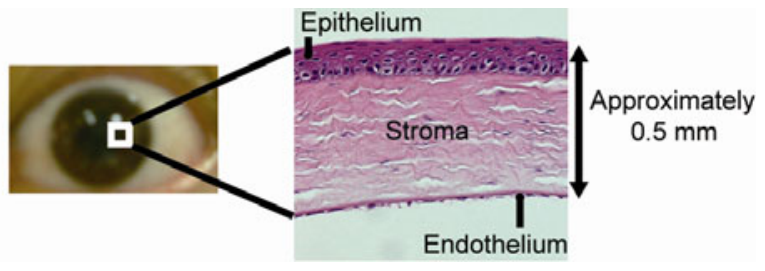

Figure 1 Composition of the cornea. The cornea is a transparent tissue with a thickness of approximately $0.5 \mathrm{~mm}$.

established yet.

Recently, regenerative medicine has been focused on in several fields as next-generation treatments for several diseases or injures, for which there is currently no cure. In the mid-1980s, transplantations of bioengineered sheet-like tissues derived from autologous epidermis to patients with severe burns [1], giant congenital nevi [2], or skin ulcers [3] were initiated as a therapeutic modality. In addition, based on a novel concept termed "tissue engineering", Langer and Vacanti [4-6] were the first to generate a bioengineered ear on the back of nude mice using biodegradable scaffolds on which chondrocyte were seeded. Subsequently, accompanied with the development of novel biomaterials used as carriers or scaffolds, such combinations have contributed to the reconstruction of a wide range of tissues (e.g. bone, arteries, blood vessels, and bladders) [7-10].

In the field of corneal reconstruction, the clinical application of regenerative medicine for denatured ocular surfaces due to severe trauma (e.g. thermal and chemical burns) or eye diseases (e.g. Stevens-Johnson syndrome, ocular pemphigoid) was firstly reported by Pellegrini et al. [11] in 1997. Such injuries or diseases lead to complete loss of corneal epithelial stem cells that govern the renewal of the corneal epithelium by generating transiently amplifying cells, which give rise to severe visual loss with corneal vascularization and opacification due to an alternative conjunctival cover on the corneal stroma [12-14]. Therefore, to reconstruct the corneal epithelium accompanied with the complementation of corneal epithelial stem cells, they developed a bioengineered corneal epithelium using autologous epithelial cells derived from the limbus, the transition area between cornea and conjunctiva, where corneal epithelial stem cells are reportedly resided $[15,16]$. Subsequently, for restoration of healthy ocular surfaces in patients, the bioengineered tissues were subjected to transplantation of the bioengineered corneal epithelium to the naked corneal stroma by surgically removing the denatured epithelium, following their harvest by treatment with the neutral protease dispase II. However, in their method, this protease treatment induced a weakness of the bioengineered tissues because proteins were digested that are involved in cell adhesion or cell-cell junctions at the basal membrane $[17,18]$, resulting in extremely difficult manipulation of the grafted tissues. In order to overcome such issues, amniotic membranes, collagen, fibrin gels, or gels cross-linked on fibron- ectin and fibrin have been utilized as carriers of bioengineered corneal epithelium [19-22]. In particular, an amniotic membrane, which has a low immunogenic potential as well as anti-inflammatory and anti-angiogenic properties, is regarded as one of majorly used carriers. However, the use of an amniotic membrane leads to decreased adhesion because the direct association between corneal epithelial cells and corneal stroma is interrupted. In addition, the negative influence of the permanently presented amniotic membrane between the reconstructed epithelium and stroma in the cornea cannot be excluded. Bioengineered corneal epithelium using fibrin gels showed improved adhesion between the reconstructed tissue and corneal stroma [19]. However, in addition to the possibility that the residual fibrin gel negatively influences optical transparency in reconstructed cornea, it is important to note that a risk of infection accompanied with the use of these biologic materials cannot be also excluded. Although there are still several issues that need to be overcome, the regenerative medicine for ocular surfaces has been steadily progressed and is regarded as one of most successful fields in the clinical application of regenerative medicine.

Previously, we established a novel method to prepare bioengineered tissues without the use of any carriers or scaffolds made from biological materials as well as protease treatments, through intelligently modifying the surface of culture dishes $[23,24]$. Our technology addresses the issues that arose from the use of biologic materials or protease treatments. Here, we introduce our novel concept of "cell sheet engineering" and regenerative therapies for corneas based on cell sheet engineering. In addition to the treatment for ocular surfaces, cell sheet engineering provides also a breakthrough in the regenerative therapy for the corneal endothelium for which clinical application have not been established yet. Consequently, our technology has greatly contributed to the progression of the regenerative medicine for corneas through the solution of traditional issues as well as the merits of carrier-free cell sheets.

\section{Cell sheet engineering based on temperature- responsive culture surfaces}

With the concept of tissue engineering based on the combination of biocompatible or biodegradable materials and living cells, the regenerative medicine has made great progress, whereas the use of scaffolds makes it very difficult to construct functional tissues with high cell density. Treatments with proteases such as dispase can be used for the construction of sheet-like tissues with high cell density without scaffolds, but render the obtained tissues fragile because of the digestion of proteins present in cell-cell junctions and extracellular matrix (ECM). In order to overcome these issues, we have proposed "cell sheet engineering" as a novel method to reconstruct tissues without both biodegradable 
scaffolds and protease treatments. The core of this approach is the application of an intelligent culture surface that is covalently-immobilized with a temperature-responsive polymer, poly( $N$-isopropylacrylamide) (PIPAAm). PIPAAm shows a hydrophobic character above its lower critical solution temperature (LCST: $32^{\circ} \mathrm{C}$ ), but reversely obtains a hydrophilic character at temperatures lower than $32^{\circ} \mathrm{C}$ through spontaneous conformational changes (Figure 2(a)). Therefore, the culture dishes on which PIPAAm is covalently immobilized at nanometer level thickness enable the control of cell adhesion only by temperature alteration [24]. In fact, under general conditions of cell culture $\left(37^{\circ} \mathrm{C}\right.$ : above LCST), the temperature-responsive surface shows an appropriate hydrophobic status, allowing various types of cells to adhere, spread, and proliferate, similar to cells cultured on normal polystyrene culture dishes (Figure 2(b)). In contrast, a reduced temperature below the LCST lets the culture dish surfaces change to a hydrophilic character, resulting in the detachment of cells by the formation of a hydration layer between the culture dish surface and the attached cells (Figure 2(b)) [24]. Therefore, following the confluent conditions of cultured cells on this intelligent surface, low-temperature treatment allows us to obtain sheet-like tissues, "cell sheets," that exhibit high cell density without the need of any scaffolds and proteases (Figure 3) [23].

(a)

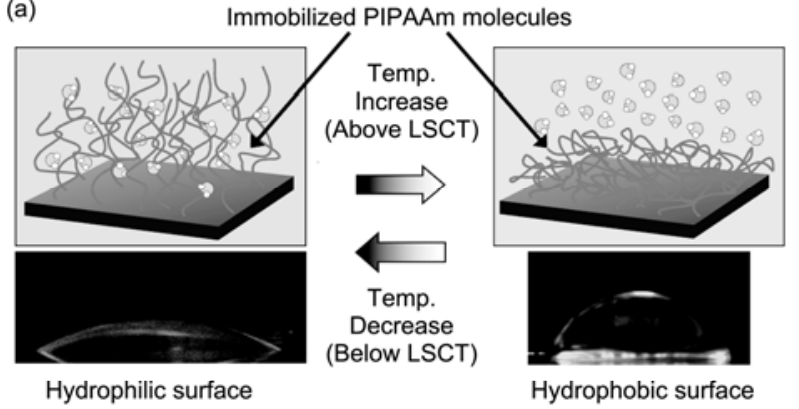

(b)
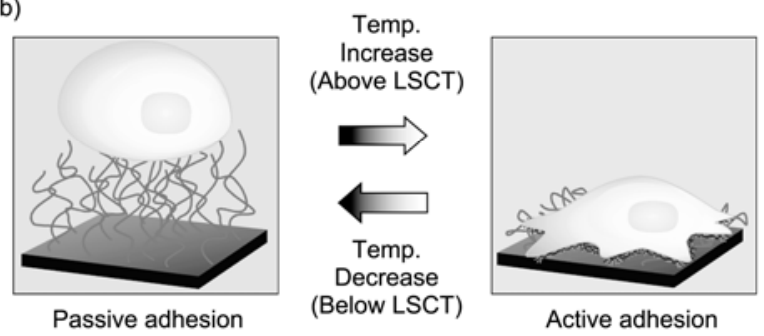

Figure 2 (Color online) Change in surface properties on temperature-responsive culture surfaces by alteration of the temperature. (a) Through conformational changes of a temperature-responsive polymer, PIPAAm, grafted on culture surfaces, our surfaces show hydrophobic properties at $37^{\circ} \mathrm{C}$ (above LSCT) but hydrophilic properties at $10^{\circ} \mathrm{C}$ (below LSCT). In addition, the wettability changes on temperature-responsive surfaces correlated with the surface properties and varied in response to the alteration of the temperature. (b) Schematic for the interactions of temperature-responsive surfaces with cells. Conditions above LSCT (e.g. $37^{\circ} \mathrm{C}$ ) allow active cell adhesion to the appropriate hydrophobic surfaces of temperature-responsive culture dishes. In contrast, at conditions below LSCT (e.g. $20^{\circ} \mathrm{C}$ ), the change to the hydrophilic property in the culture surfaces subsequently leads to the detachment of adhered cells.
Importantly, on the cells within non-invasively obtained cell sheets, membrane proteins (e.g. growth factor receptors, ion channels, and cell-to-cell junction proteins) are well sustained $[18,25]$. Therefore, these preserved surface proteins contribute to the maintenance of differentiated tissue functions in our carrier-free tissues. In particular, our cell sheets with intact cell-to-cell junction proteins and ECM showed a significantly enhanced obdurability, compared to those harvested after dispase treatment. Furthermore, maintenance of the ECM accumulated at the basal side of cells during the culture provides an enhanced adhesiveness between our cell sheets and other surfaces such as culture dishes and host tissues, which is also one of the merits of our cell sheets. So far, using temperature-responsive culture surfaces, we have established numerous types of carrier-free cell sheets derived from epidermal keratinocytes [18], renal epithelial cells [26,27], periodontal ligaments [28,29], retinal pigment epithelium [30], Thyroid cells [31], periosteal cells [32], cardiomyocytes [33,34] and oral mucosal epithelial cells $[35,36]$. In addition, we demonstrated that multilayered cell sheets enable the construction of 3-dimensional tissues with much higher cell density compared to the traditional way using biodegradable scaffolds [37-39].

On the other hand, temperature-responsive culture surfaces have been developed through the improvement of the way for the polymerization of $\mathrm{N}$-isopropylacrylamide (IPAAm) or the immobilization of PIPAAm. We originally developed temperature-responsive cell culture surface using electron-beem induced polymerization methods [40]. Other groups reported that plasma activation and plasma polymerization were also available for the surface modification with PIPPAm [41]. Recently, to precisely control chain length and density of grafted PIPAAm on the surfaces, atom transfer radical polymerization (ATRP) or reversible addition-fragmentation chain transfer (RAFT) polymerization are also used, which contributes to enhanced handling of
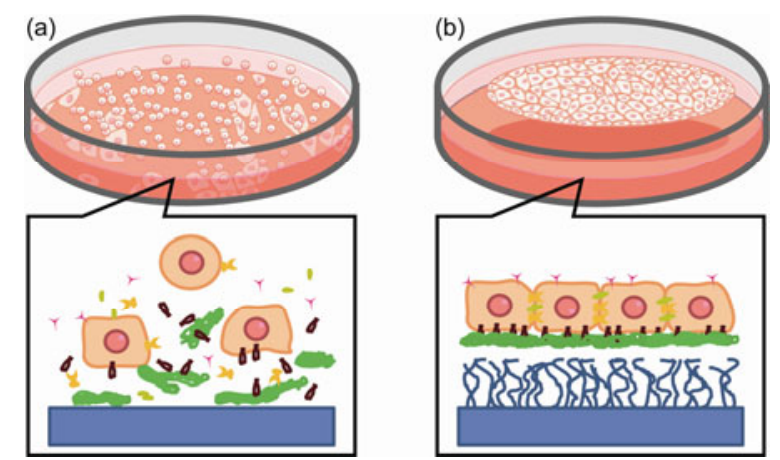

Figure 3 (Color online) Cell sheets are harvested from temperature-responsive culture surfaces by low-temperature treatment. (a) With traditional proteolytic harvest methods (e.g. trypsin or dispase), cells are detached, which is accompanied by disruption of both ECM and cell-to-cell junction proteins. (b) After the cultured cells became confluent on temperature-responsive dishes, cells can be non-invasively harvested as intact sheets with their deposited ECM maintained, by simple temperature reduction. 
cell attachment or detachment [42-46]. Furthermore, we have also developed the method to fabricate a cell sheet using the thermoresponsive surfaces that are prepared via a spin-coating method with a block copolymer consisting of PIPAAm and poly(butyl methacrylate) (PBMA) on polystyrene surfaces [47].

In addition, we have successfully demonstrate an affinity modification between cells and the culture surfaces using PIPAAm modified with cell adhesive peptide, without affecting cell detachment by thermal trigger [48]. Furthermore, the combination with micropatterning technology enables to develop cell sheets with well-controlled orientational structures $[49,50]$ and vascularized tissue grafts [51,52].

Therefore, our novel technology based on temperatureresponsive culture surfaces overcomes the issues induced by the use of proteases, biologic materials-based carrier, or biodegradable scaffolds, and greatly contributes to the construction of bioengineered tissues that are clinically safe and maneuverable in a wide range of applications in regenerative medicine.

\section{Reconstruction of the corneal epithelium}

With regard to treatments for patients with deficiency of corneal epithelial stem cells, it has been generally proposed that complementation of the alternative stem cell source is indispensable for the restoration of a healthy cornea. Traditionally, such patients were subjected to transplantation of limbal tissues derived from donor eyes as a source of stem cells [53,54]. However, limitation of donor materials and the high risk of immune rejection remain inescapable. Regenerative therapies for ocular surfaces are usually based on the transplantation of a bioengineered corneal epithelium derived from autologous limbal epithelial cells that contain stem cells, which addresses the concerns on limited donors or immune rejection. However, bioengineered tissues using biologic carriers or proteases treatment give rise to several issues related to the risk of infections, residual carriers, and fragile constructions as described above. Therefore, to overcome these issues, we have constructed carrierfree bioengineered corneal epithelium without protease treatments and biologic carriers using our temperatureresponsive culture surfaces [17]. In our method, as source of autologous corneal epithelial stem cells for patients with unilateral disease, a small biopsy sample that can be obtained from the limbus of the healthy eye has been utilized (Figure 4), similar to the previous study [11]. Following the expansion of autologous limbal epithelial cells on our generated surface, carrier-free cell sheets could be easily obtained only by low-temperature treatment and were directly transplanted to the corneal stroma from which the denatured epithelium has been removed. When the cell sheet was transferred during the transplantation, it showed superior manipulation capability compared to protease-treated

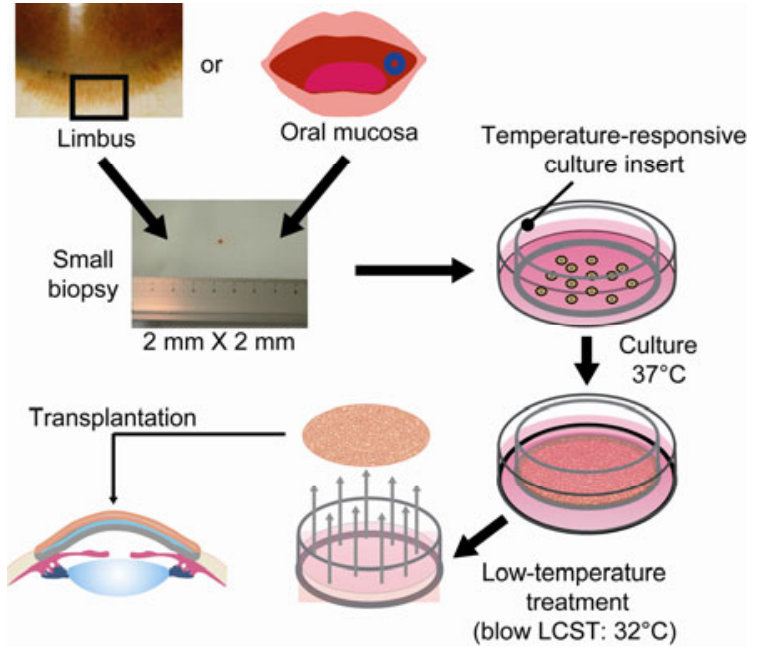

Figure 4 (Color online) Reconstruction of the corneal epithelium by using cell sheet engineering. From small biopsy samples of the limbus or oral mucosa, epithelial stem cells can be isolated and expanded on temperature-responsive culture surfaces. At the time of surgery, the fabricated epithelial cell sheets can be harvested by temperature reduction to $20^{\circ} \mathrm{C}$ for $30 \mathrm{~min}$ and transplanted directly to the host corneal stroma without the use of sutures.

tissues, which was due to enhanced obdurability achieved by preservation of the cell-cell junction or ECM [17]. In addition, the intact ECM on the basal side of cells in the cell sheets contributed to the rapid and stable attachment to the host corneal stroma, allowing stable transplantation of corneal epithelial cell sheets without the need of sutures [17]. Furthermore, it is important to note that our carrier- free cell sheets showed enhanced optical transparency compared to the bioengineered corneal epithelium using amniotic membranes or fibrin gels [17]. Thus, with regard to transplantation therapies for ocular surfaces, our technology not only contributes to overcoming traditional issues, but also provides the merits of carrier-free cell sheets harvested without protease-treatments.

However, such an advanced regenerative therapy is not available for patients with bilateral total limbal stem-cell deficiencies, because the limbus cannot be used as a cell source for bioengineered tissues. Corneal disorders induced by genetic or immune abnormalities lead to bilaterally corneal opacification in most of cases. Therefore, we focused on epithelial stem cells derived from oral mucosa as an alternative to limbal epithelial cells used in the autologous regenerative therapy of ocular surfaces (Figure 4). In fact, bioengineered tissues derived from oral mucosal epithelial cells showed similar optical transparency compared to the corneal epithelium and were available for transplantation to a rabbit corneal model [55]. Moreover, the substitution of limbal epithelial cells by oral mucosal epithelial cells is also supported by the histological observation that human oral mucosal cell sheets recapitulated a structure comparable to that of the native corneal epithelium [36]. Interestingly, in an animal model of corneal surface reconstruction, follow- 
ing the transplantation, oral mucosal epithelial cell sheets resembled an expression pattern of keratin similar to that of native corneal epithelial cells rather than native oral mucosal epithelial cells [56]. This phenotypic transformation of oral mucosal epithelial cells on the corneal stroma indicates further that these cells have the potential to be used as an appropriate substitution of limbal epithelial cells, and suggests that the interaction with corneal epithelial cells in the corneal stroma plays a key role in the maintenance of their phenotype. Therefore, our carrier-free system seems to have only a minor effect on such interactions, which may contribute to the long-maintenance of healthy conditions in transplanted cell sheets. In fact, our clinical trials using autologous oral mucosal cell sheets prepared using temperature-responsive culture surfaces have demonstrated significant improvement in visual acuity and successful long-term maintenance of healthy conditions at the ocular surface in all patients after transplantation (Figure 5) [36]. In addition, we recently established more optimized culture conditions in the preparation of cell sheets [57-59] and the validation system for tissue-engineered cell sheets $[60,61]$. Moreover, we also developed an autologous transplantation technique for patients with inherited corneal disorders by genetic modification of corneal epithelial stem cell sheets using lentiviral vectors [62]. On the one hand, we have demonstrated novel findings regarding epithelial cell biology, which may contribute to effective preparation of transplantable epithelial cell sheets [63-67]. Thus, we have further developed an application in regenerative medicine for ocular surfaces through using our technology and optimizing its associated technologies.

\section{The reconstruction of corneal endothelium}

The native corneal endothelium, a single-cell layer composed of corneal endothelial cells, resides in the proximally posterior surface of the corneal stroma, and plays a crucial role in the control of hydration and thickness of the stroma via both their ATP-dependent endothelial pumps (pump function) and focal tight junctions (barrier function), which is indispensable for the maintenance of visual acuity [68]. However, unlike corneal epithelial cells, human corneal endothelial cells are hardly capable of cell proliferation in vivo $[69,70]$. Therefore, the loss of cells in the corneal endothelium is complemented through lateral expansion in cell size by residual corneal endothelial cells, and a further decrease in the cell number leads to corneal endothelial dysfunction, resulting in the occurrence of corneal stromal edema [71]. Given these properties or functions in the corneal endothelium, we thought that our carrier-free system should be exploited in the reconstruction of the corneal endothelium, because there is only little interruption between our cell sheets and the corneal stroma. In addition, human

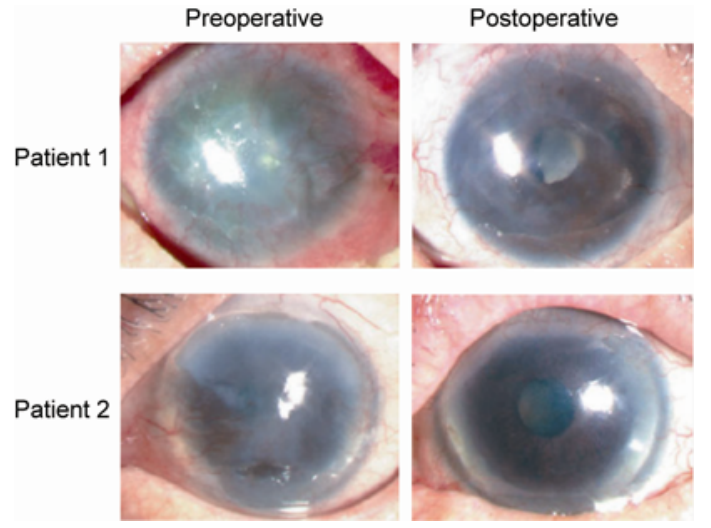

Figure 5 Eyes of patients before and after transplantation of sheets of tissue-engineered autologous epithelial cells. These photographs were taken just before transplantation of the cell sheets and postoperatively after more than 13 months. Reprinted with permission from [36], (C) 2004 Massachusetts Medical Society. All rights reserved.

corneal endothelial cells reportedly gain the potential for cell proliferation under optimized culture conditions in vitro [72-74], which enabled us to develop transplantable corneal endothelial cell sheets by using a technology that is based on temperature-responsive culture surfaces [75,76]. Furthermore, to establish a functional, bioengineered corneal endothelium, it is essential to ensure sufficient cell density in the prepared cell sheets. Therefore, in order to achieve the cell density that is observed in the healthy corneal endothelium (approximately 3000 cells $/ \mathrm{mm}^{2}$ ), corneal endothelial cells were expanded under appropriate conditions by using the explant culture and seeded onto temperatureresponsive culture surfaces at high density [75,76]. Subsequently, low-temperature treatment allowed us to obtain corneal endothelial cell sheets that recapitulated the structural and morphological characteristics observed in the native corneal endothelium, which is a single-cell layer composed of polygonal cell with both microvilli and cilia on the apical side of the cell surface. Even though it is a single-cell layer, corneal endothelial cell sheets show sufficient strength to be grasped using tweezers during the harvest, probably because of the preservation of deposited ECM, e.g. fibronectin and collagen IV [76]. Importantly, non-invasive harvest of cell sheets revealed maintenance of functional tight junctions as well as $\mathrm{Na}^{+}$and $\mathrm{K}^{+}$ATPase on cells within cell sheets [76], which play essential roles in their barrier and pump function, respectively. Indeed, our human corneal endothelial cell sheets exhibited a great potential for future clinical application, as evidenced by the observation that direct transplantation of the cell sheets to the stroma of the cornea with corneal endothelial dysfunction significantly improved corneal stromal hydration and swelling in a rabbit model (Figure 6) [75]. Although our proposed regenerative therapy for the corneal endothelium certainly demonstrated a great healing effect, cell sources for bioengineered corneal 


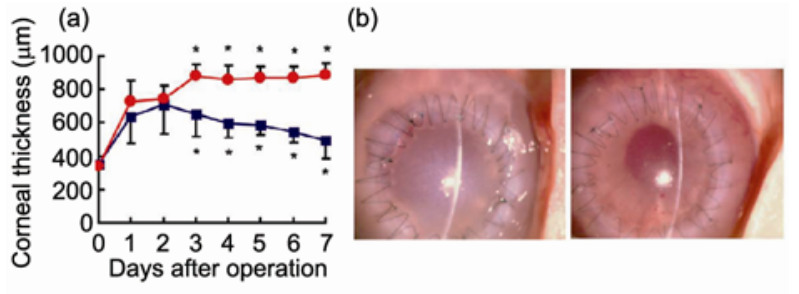

(c)

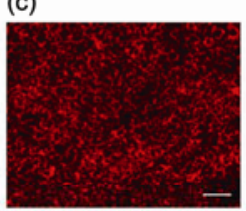

(d)
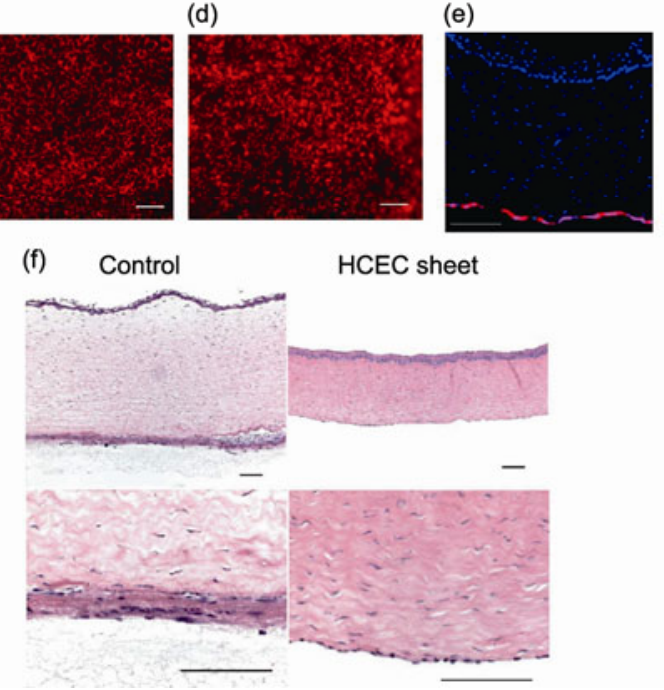

Figure 6 Functional corneal endothelial cell sheets. (a) Average corneal thickness was measured after transplantation. Control corneas (red circles); corneas receiving human corneal endothelial cell sheet transplantation (blue squares). Data represent the mean $\pm \mathrm{SD}$ and a $* P$-value of less than 0.05 was considered significant. (b) Slit lamp microscopy $7 \mathrm{~d}$ after surgery shows that the rabbit corneas receiving human corneal endothelial cell sheets were considerably more transparent, with reduced swelling. (c) Surface micrographs prior to transplantation show that DiI-labeled corneal endothelial cells are present within the tissue-engineered sheets at a similar density to that of the native corneal endothelium. (d) The $7 \mathrm{~d}$ after transplantation, DiI-derived red fluorescence is still observed at a high density in the corneas receiving corneal endothelial cell sheet transplantation. (e) Cryosections also show that the DiI-labeled corneal endothelial cell sheets remain stably attached to and survive beneath the corneal stroma. (f) Hematoxylin and eosin staining shows that the corneal endothelial cell sheets attach stably to the host stroma and there is decreased stromal swelling and cell infiltration in the corneal endothelial cell transplant group. Reprinted with permission from [75], () 2006 Federation of American Societies for Experimental Biology.

endothelial cell sheets have been hardly established so far. Unlike the corneal epithelium, ectopic cells that can serve as a functional alternative to corneal endothelial cells have not been identified yet. In addition, it is very difficult to perform a biopsy from the healthy eye safely. Therefore, induced pluripotent stem (iPS) cells are currently regarded as the most promising candidate for a cell source, whereas the clinically applicable way for differentiation of iPS to corneal endothelial cells has not been fully established yet. Thus, since our proposed therapies seem to be technically available for clinical application, the establishment of a safe cell source is indispensable to achieve further breakthroughs in the application of regenerative medicine for the corneal endothelium.

\section{Conclusion}

In the regenerative medicine for corneal epithelium, our technology based on temperature-responsive culture surfaces contributes to the solution of several issues observed in the traditional treatments (e.g. the risk of infections, residual carriers, and fragile constructions). Furthermore, in addition to patients with unilateral disorder, our advanced therapies have been available for patients with bilateral corneal stem-cell deficiencies by the use of autologous oral mucosal epithelial cells as an alternative source of stem cells except for limbal epithelial cells $[17,36]$. Importantly, our corneal epithelium cell sheets have enhanced optical transparency compared to bioengineered tissues using biologic carriers [17]. Furthermore, only marginal effects on the direct interaction with corneal stroma in the transplanted cell sheets is also one of the merits of our systems, because our previous report suggests that interaction with the host's stromal keratocytes contributes to the maintenance of unique properties of corneal epithelial cells [56]. Therefore, these advantages seem to be essential for the reconstruction of cornea. Consequently, we believe that our method greatly contributes to the progression of regenerative therapies for the corneal epithelium, and that our proposed treatments will exhibit a global dissemination as a definitive therapy for denatured ocular surfaces.

In addition, for the reconstruction of the corneal endothelium, we have developed carrier-free corneal endothelial cell sheets that exhibited significant healing effects in animal models [75,76]. Given the functions of the corneal endothelium, the advantages of our carrier-free cell sheets should be exploited in the reconstruction of the corneal endothelium. However, for the clinical application of regenerative therapies for the corneal endothelium, the establishment of a safe cell source for the bioengineered corneal endothelium is indispensable, which is currently considered the bottleneck of this therapeutic approach.

In summary, compared to the traditional techniques that use biologic carriers or biodegradable scaffolds, our method, which is based on carrier-free cell sheets without protease treatments, provides several advantages in a wide range of fields. In addition, our method is a further breakthrough in the reconstruction of the cornea, where optical transparency and interactions between each layer of the cornea are critical for the restoration of its native functions.

This work was partly supported by Creation of Innovation Centers for Advanced Interdisciplinary Research Areas Program in the Project for Developing Innovation Systems "Cell Sheet Tissue Engineering Center (CSTEC)" from the Ministry of Education, Culture, Sports, Science and Technology (MEXT), Japan.

1 Gallico G G, O'Connor N E, Compton C C, et al. Permanent coverage of large burn wounds with autologous cultured human epithelium. 
N Engl J Med, 1984, 311: 448-451

2 Gallico G G, O'Connor N E, Compton C C, et al. Cultured epithelial autografts for giant congenital nevi. Plast Reconstr Surg, 1989, 84: $1-9$

3 Phillips T J, Kehinde O, Green H, et al. Treatment of skin ulcers with cultured epidermal allografts. J Am Acad Dermatol, 1989, 21: 191199

4 Langer R, Vacanti J P. Tissue engineering. Science, 1993, 260: 920-926

5 Vacanti J P. Beyond transplantation. Third annual samuel jason mixter lecture. Arch Surg, 1988, 123: 545-549

6 Vacanti J P, Langer R. Tissue engineering: The design and fabrication of living replacement devices for surgical reconstruction and transplantation. Lancet, 1999, 354 (Suppl 1): 132-134

7 Atala A, Bauer S B, Soker S, et al. Tissue-engineered autologous bladders for patients needing cystoplasty. Lancet, 2006, 367: 12411246

8 Poh M, Boyer M, Solan A, et al. Blood vessels engineered from human cells. Lancet, 2005, 365: 2122-2124

9 Shin'oka T, Imai Y, Ikada Y. Transplantation of a tissue-engineered pulmonary artery. N Engl J Med, 2001, 344: 532-533

10 Vacanti C A, Bonassar L J, Vacanti M P, et al. Replacement of an avulsed phalanx with tissue-engineered bone. N Engl J Med, 2001, 344: 1511-1514

11 Pellegrini G, Traverso C E, Franzi A T, et al. Long-term restoration of damaged corneal surfaces with autologous cultivated corneal epithelium. Lancet, 1997, 349: 990-993

12 Buck R C. Measurement of centripetal migration of normal corneal epithelial cells in the mouse. Invest Ophthalmol Vis Sci, 1985, 26: 1296-1299

13 Kinoshita S, Friend J, Thoft R A. Sex chromatin of donor corneal epithelium in rabbits. Invest Ophthalmol Vis Sci, 1981, 21: 434-441

14 Thoft R A, Friend J. The X, Y, Z hypothesis of corneal epithelial maintenance. Invest Ophthalmol Vis Sci, 1983, 24: 1442-1443

15 Cotsarelis G, Cheng S Z, Dong G, et al. Existence of slow-cycling limbal epithelial basal cells that can be preferentially stimulated to proliferate: Implications on epithelial stem cells. Cell, 1989, 57: 201209

16 Schermer A, Galvin S, Sun T T. Differentiation-related expression of a major $64 \mathrm{~K}$ corneal keratin in vivo and in culture suggests limbal location of corneal epithelial stem cells. J Cell Biol, 1986, 103: 49-62

17 Nishida K, Yamato M, Hayashida Y, et al. Functional bioengineered corneal epithelial sheet grafts from corneal stem cells expanded $e x$ vivo on a temperature-responsive cell culture surface. Transplantation, 2004, 77: 379-385

18 Yamato M, Utsumi M, Kushida A, et al. Thermo-responsive culture dishes allow the intact harvest of multilayered keratinocyte sheets without dispase by reducing temperature. Tissue Eng, 2001, 7: 473480

19 Han B, Schwab I R, Madsen T K, et al. A fibrin-based bioengineered ocular surface with human corneal epithelial stem cells. Cornea, 2002, 21: 505-510

20 Rama P, Bonini S, Lambiase A, et al. Autologous fibrin-cultured limbal stem cells permanently restore the corneal surface of patients with total limbal stem cell deficiency. Transplantation, 2001, 72: 1478-1485

21 Schwab I R. Cultured corneal epithelia for ocular surface disease. Trans Am Ophthalmol Soc, 1999, 97: 891-986

22 Tsai R J, Li L M, Chen J K. Reconstruction of damaged corneas by transplantation of autologous limbal epithelial cells. N Engl J Med, 2000, 343: 86-93

23 Okano T, Yamada N, Okuhara M, et al. Mechanism of cell detachment from temperature-modulated, hydrophilic-hydrophobic polymer surfaces. Biomaterials, 1995, 16: 297-303

24 Okano T, Yamada N, Sakai H, et al. A novel recovery system for cultured cells using plasma-treated polystyrene dishes grafted with poly(N-isopropylacrylamide). J Biomed Mater Res, 1993, 27: 12431251

25 Kushida A, Yamato M, Konno C, et al. Decrease in culture tempera- ture releases monolayer endothelial cell sheets together with deposited fibronectin matrix from temperature-responsive culture surfaces. J Biomed Mater Res, 1999, 45: 355-362

26 Kushida A, Yamato M, Isoi Y, et al. A noninvasive transfer system for polarized renal tubule epithelial cell sheets using temperature-responsive culture dishes. Eur Cell Mater, 2005, 10: 23-30

27 Kushida A, Yamato M, Kikuchi A, et al. Two-dimensional manipulation of differentiated Madin-Darby canine kidney (MDCK) cell sheets: The noninvasive harvest from temperature-responsive culture dishes and transfer to other surfaces. J Biomed Mater Res, 2001, 54: $37-46$

28 Akizuki T, Oda S, Komaki M, et al. Application of periodontal ligament cell sheet for periodontal regeneration: A pilot study in beagle dogs. J Periodontal Res, 2005, 40: 245-251

29 Hasegawa M, Yamato M, Kikuchi A, et al. Human periodontal ligament cell sheets can regenerate periodontal ligament tissue in an athymic rat model. Tissue Eng, 2005, 11: 469-478

30 Yaji N, Yamato M, Yang J, et al. Transplantation of tissueengineered retinal pigment epithelial cell sheets in a rabbit model. Biomaterials, 2009, 3: 797-803

31 Arauchi A, Shimizu T, Yamato M, et al. Tissue-engineered thyroid cell sheet rescued hypothyroidism in rat models after receiving total thyroidectomy comparing with nontransplantation models. Tissue Eng Part A, 2009, 15: 3943-3949

32 Uchiyama $\mathrm{H}$, Yamato $\mathrm{M}$, Sasaki $\mathrm{R}$, et al. In vivo $3 \mathrm{D}$ analysis with micro-computed tomography of rat calvaria bone regeneration using periosteal cell sheets fabricated on temperature-responsive culture dishes. J Tissue Eng Regen Med, 2011, 5: 483-490

33 Shimizu T, Yamato M, Akutsu T, et al. Electrically communicating three-dimensional cardiac tissue mimic fabricated by layered cultured cardiomyocyte sheets. J Biomed Mater Res, 2002, 60: 110-117

34 Shimizu T, Yamato M, Kikuchi A, et al. Two-dimensional manipulation of cardiac myocyte sheets utilizing temperature-responsive culture dishes augments the pulsatile amplitude. Tissue Eng, 2001, 7: 141-151

35 Ohki T, Yamato M, Ota M, et al. Prevention of esophageal stricture after endoscopic submucosal dissection using tissue-engineered cell sheets. Gastroenterology, 2012, 143: 582-588

36 Nishida K, Yamato M, Hayashida Y, et al. Corneal reconstruction with tissue-engineered cell sheets composed of autologous oral mucosal epithelium. N Engl J Med, 2004, 351: 1187-1196

37 Haraguchi Y, Shimizu T, Sasagawa T, et al. Fabrication of functional three-dimensional tissues by stacking cell sheets in vitro. Nat Protoc, 2012, 7: 850-858

38 Shimizu T, Sekine H, Yang J, et al. Polysurgery of cell sheet grafts overcomes diffusion limits to produce thick, vascularized myocardial tissues. Faseb J, 2006, 20: 708-710

39 Shimizu T, Yamato M, Isoi Y, et al. Fabrication of pulsatile cardiac tissue grafts using a novel 3-dimensional cell sheet manipulation technique and temperature-responsive cell culture surfaces. Circ Res, 2002, 90: e40

40 Yamada N, Okano T, Sakai H, et al. Thermo-responsive polymeric surfaces; control of attachment and detachment of cultured cells. Makromol Chem Rapid Commun, 1990, 11: 571-576

41 Pan Y V, Wesley R A, Luginbuhl R, et al. Plasma polymerized $\mathrm{N}$-isopropylacrylamide: Synthesis and characterization of a smart thermally responsive coating. Biomacromolecules, 2001, 2: 32-36

42 Nagase K, Watanabe M, Kikuchi A, et al. Thermo-responsive polymer brushes as intelligent biointerfaces: Preparation via ATRP and characterization. Macromol Biosci, 2011, 11: 400-409

43 Takahashi H, Nakayama M, Yamato M, et al. Controlled chain length and graft density of thermoresponsive polymer brushes for optimizing cell sheet harvest. Biomacromolecules, 2010, 11: 1991-1999

44 Mizutani A, Kikuchi A, Yamato M, et al. Preparation of thermoresponsive polymer brush surfaces and their interaction with cells. Biomaterials, 2008, 29: 2073-2081

$45 \mathrm{Li} \mathrm{L}, \mathrm{Zhu} \mathrm{Y}, \mathrm{Li} \mathrm{B}$, et al. Fabrication of thermoresponsive polymer gradients for study of cell adhesion and detachment. Langmuir, 2008, 24: $13632-13639$ 
46 Xu F J, Zhong S P, Yung L Y, et al. Surface-active and stimuli-responsive polymer- $\mathrm{Si}(100)$ hybrids from surface-initiated atom transfer radical polymerization for control of cell adhesion. Biomacromolecules, 2004, 5: 2392-2403

47 Nakayama M, Yamada N, Kumashiro Y, et al. Thermoresponsive poly(N-isopropylacrylamide)-based block copolymer coating for optimizing cell sheet fabrication. Macromol Biosci, 2012, 12: 751-760

48 Ebara M, Yamato M, Aoyagi T, et al. The effect of extensible PEG tethers on shielding between grafted thermo-responsive polymer chains and integrin-RGD binding. Biomaterials, 2008, 29: 3650-3655

49 Takahashi H, Nakayama M, Shimizu T, et al. Anisotropic cell sheets for constructing three-dimensional tissue with well-organized cell orientation. Biomaterials, 2011, 32: 8830-8838

50 Takahashi H, Nakayama M, Itoga K, et al. Micropatterned thermoresponsive polymer brush surfaces for fabricating cell sheets with well-controlled orientational structures. Biomacromolecules, 2011, 12: $1414-1418$

51 Muraoka M, Shimizu T, Itoga K, et al. Control of the formation of vascular networks in 3D tissue engineered constructs. Biomaterials, 2013, 34: 696-703

52 Tsuda Y, Shimizu T, Yamato M, et al. Cellular control of tissue architectures using a three-dimensional tissue fabrication technique. Biomaterials, 2007, 28: 4939-4946

53 Kenyon K R, Tseng S C. Limbal autograft transplantation for ocular surface disorders. Ophthalmology, 1989, 96: 709-722

54 Tsubota K, Satake Y, Kaido M, et al. Treatment of severe ocular-surface disorders with corneal epithelial stem-cell transplantation. N Engl J Med. 1999, 340: 1697-1703

55 Nakamura T, Endo K, Cooper L J, et al. The successful culture and autologous transplantation of rabbit oral mucosal epithelial cells on amniotic membrane. Invest Ophthalmol Vis Sci, 2003, 44: 106-116

56 Hayashida Y, Nishida K, Yamato M, et al. Ocular surface reconstruction using autologous rabbit oral mucosal epithelial sheets fabricated ex vivo on a temperature-responsive culture surface. Invest Ophthalmol Vis Sci, 2005, 46: 1632-1639

57 Takagi R, Yamato M, Murakami D, et al. Preparation of keratinocyte culture medium for the clinical applications of regenerative medicine. J Tissue Eng Regen Med, 2011, 5: 63-73

58 Murakami D, Yamato M, Nishida K, et al. The effect of micropores in the surface of temperature-responsive culture inserts on the fabrication of transplantable canine oral mucosal epithelial cell sheets. Biomaterials, 2006, 27: 5518-5523

59 Murakami D, Yamato M, Nishida K, et al. Fabrication of transplantable human oral mucosal epithelial cell sheets using temperature-responsive culture inserts without feeder layer cells. J Artif Organs, 2006, 9: 185-191

60 Takagi R, Yamato M, Murakami D, et al. Fabrication and validation of autologous human oral mucosal epithelial cell sheets to prevent stenosis after esophageal endoscopic submucosal dissection. Pathobi- ology, 2011, 78: 311-319

61 Hayashi R, Yamato M, Takayanagi H, et al. Validation system of tissue-engineered epithelial cell sheets for corneal regenerative medicine. Tissue Eng Part C Methods, 2010, 16: 553-560

62 Watanabe K, Yamato M, Hayashida Y, et al. Development of transplantable genetically modified corneal epithelial cell sheets for gene therapy. Biomaterials, 2007, 28: 745-749

63 Kondo M, Yamato M, Takagi R, et al. The regulation of epithelial cell proliferation and growth by IL-1 receptor antagonist. Biomaterials, 2013, 34: 121-129

64 Takagi R, Yamato M, Kushida A, et al. Profiling of extracellular matrix and cadherin family gene expression in mouse feeder layer cells: Type VI collagen is a candidate molecule inducing the colony formation of epithelial cells. Tissue Eng Part A, 2012, 18: 2539-2548

65 Obokata H, Yamato M, Tsuneda S, et al. Reproducible subcutaneous transplantation of cell sheets into recipient mice. Nat Protoc, 2011, 6: 1053-1059

66 Sugiyama H, Maeda K, Yamato M, et al. Human adipose tissue-derived mesenchymal stem cells as a novel feeder layer for epithelial cells. J Tissue Eng Regen Med, 2008, 2: 445-449

67 Umemoto $\mathrm{T}$, Yamato M, Nishida $\mathrm{K}$, et al. Limbal epithelial side-population cells have stem cell-like properties, including quiescent state. Stem Cells, 2006, 24: 86-94

68 Dikstein S, Maurice D M. The active control of corneal hydration. Isr J Med Sci, 1972, 8: 1523-1528

69 Murphy C, Alvarado J, Juster R, et al. Prenatal and postnatal cellularity of the human corneal endothelium. A quantitative histologic study. Invest Ophthalmol Vis Sci, 1984, 25: 312-322

70 Svedbergh B, Bill A. Scanning electron microscopic studies of the corneal endothelium in man and monkeys. Acta Ophthalmol, 1972, 50: 321-336

71 Kaufman H B B, McDonald M. The Cornea. 2nd ed. Woburn, MA: Butterworth-Heinemann, 1998

72 Egan C A, Savre-Train I, Shay J W, et al. Analysis of telomere lengths in human corneal endothelial cells from donors of different ages. Invest Ophthalmol Vis Sci, 1998, 39: 648-653

73 Senoo T, Joyce N C. Cell cycle kinetics in corneal endothelium from old and young donors. Invest Ophthalmol Vis Sci, 2000, 41: 660-667

74 Wilson S E, Weng J, Blair S, et al. Expression of E6/E7 or SV40 large $\mathrm{T}$ antigen-coding oncogenes in human corneal endothelial cells indicates regulated high-proliferative capacity. Invest Ophthalmol Vis Sci, 1995, 36: 32-40

75 Sumide T, Nishida K, Yamato M, et al. Functional human corneal endothelial cell sheets harvested from temperature-responsive culture surfaces. Faseb J, 2006, 20: 392-394

76 Ide T, Nishida K, Yamato M, et al. Structural characterization of bioengineered human corneal endothelial cell sheets fabricated on temperature-responsive culture dishes. Biomaterials, 2006, 27: 607614

Open Access This article is distributed under the terms of the Creative Commons Attribution License which permits any use, distribution, and reproduction in any medium, provided the original author(s) and source are credited. 